Please do not remove this page

RMIT

UNIVERSITY

\title{
Framework for improving workflow stability: deployment of optimized capacity buffers in a synchronized construction production
}

Arashpour, Mehrdad; Wakefield, Ronald; Blismas, Nick; Lee, Eric

https://researchrepository.rmit.edu.au/esploro/outputs/9921862071601341/filesAndLinks?institution=61RMIT_INST\&index=null

Arashpour, M., Wakefield, R., Blismas, N., \& Lee, E. (2014). Framework for improving workflow stability:

deployment of optimized capacity buffers in a synchronized construction production. Canadian Journal of Civil Engineering, 41(12), 995-1004. https://doi.org/10.1139/cjce-2014-0199

Document Version: Accepted Manuscript

Published Version: https://doi.org/10.1139/cjce-2014-0199

Repository homepage: https://researchrepository.rmit.edu.au

(C) 2014 Canadian Science Publishing

Downloaded On 2023/04/26 19:32:12 +1000 
Thank you for downloading this document from the RMIT Research Repository.

The RMIT Research Repository is an open access database showcasing the research outputs of RMIT University researchers.

RMIT Research Repository: http://researchbank.rmit.edu.au/

\section{Citation:}

Arashpour, M, Wakefield, R, Blismas, N and Lee, E 2014, 'Framework for improving workflow stability: deployment of optimized capacity buffers in a synchronized construction production', Canadian Journal of Civil Engineering, vol. 41, no. 12, pp. 995-1004.

See this record in the RMIT Research Repository at:

https://researchbank.rmit.edu.au/view/rmit:28951

Version: Accepted Manuscript

Copyright Statement: (c) 2014 Canadian Science Publishing

Link to Published Version:

http://dx.doi.org/10.1139/cjce-2014-0199 


\section{3}

4

\begin{abstract}
Construction sites are dynamic environments due to the influence of variables such as changes in design and processes, unsteady demand, and unavailability of trades. These variables adversely affect productivity and can cause an unstable workflow in the network of trade contractors. Previous research on workflow stability in the construction and manufacturing domains has shown the effectiveness of 'pull' production or 'rate driven' construction. Pull systems authorize the start of construction when a job is completed and leaves the trade contractor network. However, the problem with pull systems is that completion dates are not explicitly considered and therefore additional mechanisms are required to ensure the due date integrity. On this basis, the aim of this investigation is to improve the coordination between output and demand using optimal-sized capacity buffers. Towards this aim, production data of two Australian construction companies were collected and analysed. Capacity and cost optimizations were conducted in order to find the optimum buffer that strikes the balance between late completion costs and lost revenue opportunity. Following this, simulation experiments were designed and run in order to analyse different 'what-if' production scenarios. The findings show that capacity buffers enable builders to ensure a desired service level. Size of the capacity buffer is more sensitive to the level of variability in contractor processes than other production variables. This work contributes to the body-of-knowledge by improving production control in construction and deployment of capacity buffers in order to achieve a stable workflow. In addition, construction companies can use the easy-to-use framework tested in this study in order to compute the optimal size for capacity buffers that maximizes profit and prevents late completions.
\end{abstract}

Keywords: Analytical modeling; Capacity buffer; Construction; Cost optimization; Discrete event simulation; Productivity; Pull workflow; Queue; Time series analysis; Under-capacity planning and scheduling

\title{
1. Introduction
}

Production in dynamic environments such as construction sites are prone to variability caused by external factors such as unsteady demand and also internal factors such as unavailability of resources. This high level of variability results in late completions, decreased output, and lost revenue opportunity for contractors (Lee and Diekmann 2011, Chanmeka, Thomas et al. 2012). Stabilising the workflow in the trade contractor network coordinates the production output and demand and results in a synchronised production. Prior work in the construction literature has focused on designing and implementing pull production systems in order to stabilise the workflow in construction production systems (Im, Han et al. 2009).

The main workflow control mechanism in pull production or rate driven construction is to maintain a constant work-in-process (CONWIP) for the trade network over the production period. The CONWIP protocol enables trade contractors to plan ahead in order to accommodate the demand. Since due dates are not explicitly considered in pull systems, a second control mechanism is required. A capacity buffer or intentional under-capacity scheduling ensures due date integrity in the pull production (Hopp and 
Spearman 2008). However, as stated by González, Alarcón et al. (2011), research on capacity buffers and their effects on tangible performance measures in the construction literature is sparse.

This paper aims to improve the coordination of demand and output of construction using an optimal capacity buffer. In order to achieve this, production data of two Australian construction companies were collected. Then, time series analysis was used to analyse the data and find the production capacity for a future production period. In the next step, capacity and cost optimizations were conducted in order to find the optimal capacity buffer that strikes the balance between late completions and lost revenue opportunity. Finally, results of the mathematical modelling were linked to a discrete event simulation engine where 1200 simulation experiments were designed and run in order to analyse production scenarios in the real-life construction. The findings clearly show that loading the network of trade contractors to full capacity is not always the most profitable policy. In fact, workflow in the network of trade contractors can be stabilised using optimal-sized capacity buffers. Furthermore, the tested and validated framework could be adopted by builders in order to maximise the profit and avoid late completion costs.

\section{Background}

The prevalence of schedule overruns in the building industry is high (Kim 2009). As the common practice in the industry, risk of late completion is transferred to trade contractors by linking remunerations to the completion of processes. Any remaining risk is then transferred to clients by minimising late completion penalties in the building contractual terms. However, the manufacturing industry has dealt with schedule overruns in a more robust way. Initiatives such as the Toyota Production System (TPS) have tried to continually improve the production environment (Lander and Liker 2007). Furthermore, workflow control protocols such as 'Kanban' attempt to stabilise the workflow in the plant as much as possible and reduce the probability of schedule overruns.

Variability in the production environment will result in late completions regardless of how much the environment has been improved. Variation is the common cause of lack of alignment between demand and production in the construction industry and reducing it should be the top priority to builders (Leaman and Bordass 1999). In the construction industry, there are numerous opportunities to reduce variation. Firstly, variability in building processes can be minimized by adopting flow-smoothing techniques. These include but are not limited to using pull workflow, standardizing construction operations, conducting effective inspections and managing construction rework. Secondly, variation inside the trade contractor network is reduced when builder maintains a long-term working relation with them. In this way, there is guarantee that the builder gets the specialty trades when it needs them. Furthermore, trade contractors will also adopt variability reduction techniques suggested to them by the builder. Overall, reducing variation is pivotal to improving the performance in construction projects over time.

In the following sections, two production planning approaches in the volume house building sector are presented.

\subsection{Due date driven house construction}

77 In the traditional construction management approach, building new homes are initiated by signing new 
subcontractors are able to catch up with that. However, during construction boom periods, when demand exceeds supply, this approach is not effective. During boom periods, pushing new jobs into the interconnected network of trade, creates numerous unfinished jobs and workflow congestion. In other words, trade contractors, as the main labour resource in the production network, will be fully utilized and therefore unfinished jobs queue up, waiting for the first resource to become available (Damrianant and Wakefield 2000).

Another problem for achieving due date integrity in push construction is caused by the ubiquity of variability and uncertainty in construction worksites. There are many sources of variability in construction sites such as quality problems and rework (Fayek, Dissanayake et al. 2004, Hegazy and Menesi 2012, Hazini, Dehghan et al. 2013), changes in design and processes (Thomas, Lee et al. 2008), labour productivity (Sonmez 2007, Jarkas 2010, Arashpour, Shabanikia et al. 2012, Dai and Goodrum 2012), contractor's cash flow (Son, Mack et al. 2006, Zayed and Nosair 2006) and undesirable weather conditions (Moselhi, Gong et al. 1997, Shahin, Abourizk et al. 2014). Variability prevents a stable and smooth workflow in the construction network and downgrades the performance measures such as completion time and throughput (Hewage, Gannoruwa et al. 2011).

\subsection{Rate driven house construction}

In order to rectify the problems of due date driven production, rate driven construction focuses on stability of workflow in the interconnected network of trades and authorises new constructions only when a 'void' in the workflow becomes available upon the completion of a house. In this way, the house production network does not become congested as new starts are only authorised upon the availability of resources. This workflow management strategy, which is very similar to pull production in the manufacturing industry, has been successfully tested in large construction projects (Bashford, Sawhney et al. 2003). Using a capacity buffer in dealing with unscheduled contingencies enables rate driven construction to effectively address variability in construction sites (Arashpour, Wakefield et al. 2013).

Rate driven construction offers significant benefits over due date driven approaches. To mention some benefits, rate driven production systems are more efficient, more robust to control errors, and more supportive of improving quality (Ballard and Koskela 2009). Furthermore, setting an optimal production level (quota) with an appropriately sized capacity buffer can result in coordination between output and demand.

\subsection{Using capacity buffers}

Traditional methods of project planning and scheduling such as critical path method (CPM) are driven by critical events and do not explicitly consider the production rate of construction networks. New management approaches such as lean construction (Ballard 2000) and the critical chain project management (Goldratt and Cox 2005) propose using capacity buffers in order to address the variability in the production environment. Oversized buffers in construction projects can be wasteful, hinder performance and disrupt the workflow (Horman and Thomas 2005). Undersized buffers, on the other hand, increase the risk of late completions and a poor service level. Performance of a production system is measured by its service level $(S L)$ that shows the percentage to which production targets have been achieved (Sezer and Bröchner 2013). In construction projects, $S L$ can be defined as the percentage of ontime and on-budget delivery. The current research aims to develop a framework to find optimal-sized capacity buffers. 
The probability of missing the production target should be reasonably low so as to avoid frequent late completions. Consequently, trade-offs need to be made in order to set an optimal production target because high production levels increase the risk of schedule overruns and therefore costs of a late completion. On the other hand, low production levels or under capacity scheduling result in a profit loss because of missing sales opportunities. The research proposes a framework that realizes this trade-off and sets an optimal capacity buffer to improve workflow stability.

\section{Research method}

\subsection{Theoretical basis of the framework}

The purpose of this investigation is to find an optimal capacity buffer that maximises the builder profit by stabilising the workflow and minimising late completion costs. Although the theoretical basis of the proposed framework to achieve this purpose has been partly adopted from quota setting research in the manufacturing industry, it has been customised in order to reflect realities in the construction production. High levels of variability, on-going site establishment costs, late completion penalties, and different whatif scenarios in construction are among the factors considered in structuring the framework. Fig. 1 illustrates the proposed framework in this research.

\section{Fig. 1. Framework for improving the workflow stability using an optimized capacity buffer}

\subsection{Stages of the framework}

As can be seen in Fig.1, the framework proposes the following four stages.

Stage 1- Collecting the production data: Important information reflecting the production network capacity should be recorded. Some data reflect the production rate such as number of houses started and completed per month. Furthermore, degree of the workflow stability is reflected by the standard deviation of time between completions. These data points will enter the computations in next stages of the framework.

Stage 2- Computing the gross production capacity of the network: Having collected the actual production data, an average production capacity for the construction network can be computed. Since factors affecting the construction demand, and consequently production, such as house design, market competition, and builder's own marketing are persistent over time, past data can be indicative of future and time series can serve as a suitable tool for finding a gross production capacity (Choy and Ruwanpura 2006, Dissanayake and Fayek 2008, Lee, Fung et al. 2013). This gross production capacity can facilitate management of the construction workflow in the following stages.

Stage 3- Setting an optimal capacity buffer: This part of the framework addresses minimising the probability of late completions by setting a properly sized capacity buffer. Towards this aim, analytical models (see Equations [4] to [6]) are used to formally state the problem of finding the optimal production level and capacity buffer in the construction production. In stage three, two scenarios are analysed. In the first scenario, there is no significant late completion cost for the builder and the major concern is the capacity of the trade contractor network. In the second scenario, late completion costs are significant. Therefore, both capacity and cost optimizations are conducted in order to find the optimal capacity buffer. 
Stage 4- Real time simulation of what-if scenarios: The results of optimization modelling in stage three are linked to a discrete event simulation engine where simulation experiments are designed and run in order to analyse different what-if scenarios in the construction production. Simulation results are recorded in an output data file and can be updated upon the emergence of new production scenarios.

In terms of applying the framework in a construction setting, results of the framework can be automatically used for setting production levels. Actual on-site progress can be used for reconsidering the size of the capacity buffer in a future production period. Iterative processes of the framework can be repeated in short time intervals in order to have a more accurate production control.

\section{Results}

\subsection{Stage 1- Collecting the production data}

This research used a systematic approach for data collection that is illustrated in Fig.2.

\section{Fig. 2. Process of data collection and analysis in the current research}

In order to conduct the analysis in the second stage of the framework, number of house completions and standard deviation of time between completions were recorded (from January 2011 to December 2013). Production data were collected in standard production units, where a medium-size one-story house was considered as the standard unit. Multi-story/big and small houses were accommodated in the statistical analysis as a multiple/submultiple of the standard production unit (e.g. 1.2x or 0.8x). Number of monthly completions fluctuated between 26 and 54 houses over this production period. Availability of data over long periods of time increases the precision and reliability of predictive models (Blair, Lye et al. 1993).

\subsection{Stage 2- Finding the gross production capacity of the trade contractor} network

In order to predict the gross production capacity of the trade network in the next production period, four time series forecasting models were used to analyse the data: moving average, single exponential smoothing, double exponential smoothing and the Winter's method. These models predict the gross production capacity by using smoothing constants, $\alpha, \beta$ and $\gamma$. Care was taken in order to automate different stages of the framework and minimise the required user interference. For example, Solver, the internal optimization tool in MS Excel, was used to compute the optimum values for smoothing constants. In order to compare forecasting models, three quantitative measures were used: mean absolute percentage error (MAPE), mean absolute deviation (MAD), and mean square deviation (MSD). These accuracy measures were computed using Eq. 1 to 3.

[1] $\quad$ MAPE $=\frac{\sum_{t=1}^{n} \mid\left(x_{t}-\hat{x}_{t)} / x_{t} \mid\right.}{n} \times 100$

[2] $\quad M A D=\frac{\sum_{t=1}^{n}\left|x_{t}-\hat{x}_{t}\right|}{n}$

[3] $\quad M S D=\frac{\sum_{t=1}^{n}\left[x_{t}-\hat{x}_{t}\right]^{2}}{n}$ 
In Equations 1 to 3, $x_{t}$ is the actual number of monthly completions, $\hat{x}_{t}$ is the gross capacity forecast and $n$ is the number of observations, which is 36 (months) in this investigation. Each of the accuracy measures computes a numerical score for the difference between actual and fitted values. Smaller values of accuracy measures show a greater forecasting precision. Table 1 presents the accuracy measures for the four predictive models.

\section{Table 1 Three quantitative measures for evaluating the accuracy of gross capacity forecasting}

Comparing the measures of precision in table 1, the Winter's method has the smallest accuracy measure values and therefore is the most accurate model to find the gross production capacity of the trade contractor network. This is because the Winter's method captures seasonality and does not overshoot or undershoot the actual production data. Therefore this study uses the Winter's forecasting model and Fig. 3 shows the results of this model for gross capacity analysis over the coming production period.

\section{Fig. 3. Gross production capacity of the trade contractor network (house/month)}

A reasonably accurate capacity forecast based on the actual production records enables builders to plan ahead and find the most cost-effective way to operate their production network. For example, as Fig. 3 shows, the gross production capacity forecast for the coming month is equal to 42 houses and therefore the network of trades is orchestrated so that this level of monthly production can be achieved. That is, the monthly productivity mean or gross production capacity of the trade network is set to $\mu=42$.

Results of the second stage are used to set periodic production targets. Actual on-site progress provides input for reconsidering targets in a future production period. Iterative processes of the framework are repeated in one-month intervals in this research to adjust periodic production targets frequently and have a more accurate production control.

However, actual number of house completions is often less than the gross capacity of the trade network because of the usual contingencies such as unavailability of trade contractors, quality problems and rework, and inclement weather conditions (Arashpour and Arashpour 2010). Actual house completion times are inflated dependent on the presence of variability/uncertainty and so is the risk of undergoing extra costs such as on-going site establishment costs and late completion penalties.

In order to minimise the probability of late completions and stabilise the workflow within the trade contractor network, an optimal-sized capacity buffer is required. In the next sections, two different analytical models are developed to find the optimal size of the capacity buffer in two production scenarios. In the first scenario, late completion costs are not significant for the builder and decision on the size of the capacity buffer is based on the trade network capacity. In the second scenario, however, late completion penalties and on-going site establishment costs are considerable and both capacity and cost optimizations are conducted. 


\section{3a. Stage 3- Setting the capacity buffer based on the capacity of the trade} contractor network (scenario 1)

In order to find an optimal capacity buffer, this scenario assumes that late completion costs are not significant and decision making is based on the capacity of the trade network. If the agreed completion date is not met, the builder undergoes extra costs associated with a late completion. In this scenario, setting the work-in-process (WIP) level is the most important control measure. In addition, another control measure is also required in order to buffer variability and coordinate the construction output with due dates (Hsie, Chang et al. 2009, Arashpour, Wakefield et al. 2013).

The capacity of a house building network depends on both mean and standard deviation of production. Level of workflow stability in the interconnected network of trades can be reflected by standard deviation of time between completions (Koskela, Sacks et al. 2012). For instance, two builders may have identical production capacity average $(\mu)$ but standard deviation of time between completions $(\sigma)$ is greater in the production network of the first builder (see Fig. 4a). Understandably, the second builder needs a smaller capacity buffer in order to accommodate a similar demand level. In other words, the production predictability of the second builder is more than the first one.

\section{Fig. 4a. Standard deviation of time between completions (Workflow stability) Fig. 4 b.Capacity buffer (92\% service level)}

\section{Fig. 4. Production curves of construction networks}

Fig. $4 \mathrm{~b}$ shows that production processes can be approximated by normal distribution with a mean that is equal to the gross production capacity (calculated at stage 2). As illustrated, the network of trade contractors has the gross capacity of completing 42 houses per month. The first control measure in the rate driven (pull) environment controls the work-in-process (WIP) level so that no more than 42 houses are started monthly. The second control measure (capacity buffer) ensures that start and finish of houses are coordinated and production synchronisation is maintained. For example, a capacity buffer of seven houses in Fig. 4b, coordinates the number of starts and completions in $92 \%$ of time. In other words, the probability of missing a target house completion (THC) or quota of 35 is only 8\% (highlighted area on the graph) and the builder achieves a service level $(S L)$ of $92 \%$. Production curves of builders with lower variability in production have thinner tails. Therefore, probability of missing the target house completion will become less (see Fig. 4a). Assuming a normal distribution for the house building processes, the problem of finding an optimal capacity buffer can be formulated as Eq.4.

$$
\Phi\left(\frac{T H C-\mu}{\sigma}\right)=1-S L
$$

In Equation 4, $\Phi($.$) is the cumulative distribution function (CDF) of production, THC is the target house$ completion and $S L$ is the service level aimed by the house builder (reliability degree of production). The capacity buffer is equal to $T H C-\mu$. The capacity buffer adopts negative values as it downsizes the gross production capacity by a safety factor. For example, suppose that the trade contractor network has the 
gross weekly production capacity of 11 houses with a standard deviation of two. If the desired service level is 85\%, the model expressed by Equation 4 will return a target house completion (THC) of 9. Therefore, the capacity buffer is equal to $(-2)$ houses or $18 \%$ of the gross production capacity. In other words, by adopting this capacity buffer, the builder will be able to coordinate dates of house start and finish and synchronise production in $85 \%$ of time. It is worth mentioning that all computations can be automated using built-in functions in standard statistical packages or MS Excel. For example, in order to return the standard normal distribution, NORM.S.DIST(.) in Excel 2010 and PHI(.) function in Excel 2013 were used.

Table 2 presents $T H C$ values for different service levels of 85, 90 and 95\%. The size of the capacity buffer is equal to $T H C-\mu$ in each construction production scenario.

Table 2 Capacity buffer in 18 production scenarios with different service levels

As can be seen in table 2, larger values of gross production capacity return higher $T H C$. However, $T H C$ is decreased by either increasing the standard deviation of time between completions or the desired service level. These results are in line with those of Han, Hong et al. (2011), highlighting the importance of variability buffering in construction processes, especially when higher service levels are desired.

Fig. 5 plots increasing size of the capacity buffers against standard deviations of time between completions, which measures the level of workflow stability.

\section{Fig. 5. Upward trend in the size of the capacity buffer resulted by increasing the standard deviation of} time between completions

The linear relationship between the level of workflow stability and the size of capacity buffers is the output of the optimisation model expressed by Equation 4. A more realistic non-linear relationship that is plotted by the outputs of the fourth stage of the framework will be illustrated in Fig. 8b. The model expressed by Equation 4 can be used when capacity of the trade network is the main independent variable affecting the construction production. However, upon the existence of significant late completion costs, this factor should also enter the decision making process on the capacity buffer. In such situations, builders need to consider the trade-off between a bigger capacity buffer, which imposes the lost revenue opportunity, and a smaller buffer, which increases the late completion costs. The model developed in the next section, realizes this trade-off and optimizes the size of the capacity buffer accordingly.

\section{3b. Stage 3- Setting the optimal size of capacity buffers based on both production} capacity and late completion costs (scenario 2)

In order to find an optimal size for capacity buffers, major costs associated to a late completion should be considered and decision making is based on both these costs and capacity of the trade network.

In order to assist developing a specific model for setting the optimal capacity buffer, the gross production capacity was computed in the second stage of the framework, which is equal to 42 houses per month or 
almost 10 houses per week. If agreed completion dates are not met, builders have to pay extra costs of ongoing site establishment and late completion penalties, based on the house building contract. Consider the total cost of late completion $\left(C_{L C}\right)$ to be $\$ 300$ per week for each house. So for a builder with 10 houses under construction, $C_{L C}=\$ 3000$ for a week of delay in completion.

Let the net profit of the builder per house be ' $e$ ' and the total expected earnings (net revenue minus expected $C_{L C}$ ) be denoted by ' $E$ '. In this way, the problem of finding an optimal capacity buffer can be formulated as Equation [5].

$$
\max _{T H C} E=e \times T H C-C_{L C} \times p(\text { late completion })
$$

In Equation [5], ' $p$ ' is the probability of having a late completion and size of the capacity buffer is equal to $T H C-\mu$. The optimization problem is to find an optimal buffer that strikes the economic balance. While increasing buffer size affects the objective function by causing lost sales, decreasing buffer size affects the objective function by increasing the probability of late completions and associated costs. Assuming a normal distribution for production with mean $\mu$ and standard deviation $\sigma$, the capacity buffer can be expressed as $T H C-\mu=-m \sigma$. Now the decision variable becomes $m$ and we need to find out how many standard deviations below $\mu$ the capacity buffer should cover. In a similar approach to Hopp, Spearman et al. (1993), the problem was formulated as Equation [6].

$$
\max _{T H C} E=e(\mu-m \sigma)-C_{L C} \times p[1-\Phi(m)]
$$

In Equation 6, $\Phi($.$) represents the cumulative distribution function of production. A unique solution to$ Equation 6 can be yielded by differentiating the objective and setting it equal to zero,

$$
m^{*}=\left[2 \ln \left(\frac{C_{L C}}{\sqrt{2 \pi} \sigma e}\right)\right]^{1 / 2}
$$

The optimal value of $m$ in Equation 7 is used to compute the optimal capacity buffer size.

$$
T H C^{*}-\mu=-m^{*} \times \sigma
$$

Equation 8 shows that both gross capacity mean and standard deviation of time between completions affect the size of capacity buffer. For example, in a case in which the net profit for building a unit is $\$ 43500$ and it takes 6 months to complete the unit, the potential weekly profit of the builder will be $\$ 1670$. Assuming the sum of site establishment costs and late completion penalties to be $\$ 400$ per week, the total $C_{L C}$ will be $\$ 4000$ for 10 units under construction. Plugging these values into the above model returns the optimal THC or production quota of 8.0 and optimal capacity buffer of (-2) houses. This capacity buffer, which is equal to $20 \%$ of the gross production capacity, can maximise the profit of the builder in this production scenario.

Table 3 shows how these two variables change the house completion target (THC) and consequently the capacity buffer size. As stated earlier, all computations were automated using built-in functions and the internal optimization tool in MS Excel. 
Results in table 3 can be used to find the optimal capacity buffer. For example, in a case where the trade contractor network has a gross production capacity of 10 houses per week with a standard deviation of one house and weekly cost of late completion is $\$ 400$, the model returns a THC equal to 8.4. This capacity is equal to $16 \%$ of the gross production capacity of the trade network.

Results of the optimization model show that increasing the standard deviation of production and costs of late completion $\left(C_{L C}\right)$ decreases the target house completion or production quota. This is consistent with findings of Georgy (2008), indicating the impact of variability in production on the workflow stability and required capacity buffers. In the next phase of the framework, comprehensive models with more stochastic variables can be analysed using Monte Carlo simulation.

\subsection{Stage 4- Real-time simulation of what-if scenarios}

Data obtained in previous studies has shown that variability in the processes of trade contractors results in a reduced throughput, low resource utilisation levels and a higher allocation of overheads (Tommelein, Riley et al. 1999). Construction sites are dynamic environments and production in such an environment is subject to numerous nondeterministic variables. In order to conduct a real-time analysis of these stochastic variables, simulation experiments were designed and run as construction projects are not appropriate laboratories for multiple replications in quantitative studies (AbouRizk, Halpin et al. 2011).

Using the collected production data from the two construction companies, a total of 1200 simulation experiments were designed. Different variables were used in the models, including five gross production capacities, six standard deviations of time between completions, four service levels and 10 values for late completion costs. The project workflow was simulated using the ARENA discrete-event simulation software. Interested readers can refer to Arashpour, Wakefield et al. (2014) for a more detailed treatment of the modelling approach. Input variables to simulation models were automatically read from an excel spread sheet that contained the collected data.

Histograms of the collected data points were plotted and best-matching probability distributions were fit to the data. Selected probability distributions were evaluated against three goodness-of-fit tests; Anderson-Darling test, Kolmogorov-Smirnov test, and Chi-Square test. Care was taken in order to construct the simulation model so that it reflects the reality in the real-world construction setting. For example, the traditional Poisson process with exponential inter-arrival time was not used for representing random arrival of jobs to the construction network. As illustrated in Fig. 6, the three goodness-of-fit tests suggest that random arrivals can best be represented by Gamma distribution with the shape factor of 2.533 and scale factor of 5.999 .

\section{Fig. 6. Histogram of collected data on inter-arrival times}

Best-fitting probability distributions were used in order to feed the simulation model with the knowledge extracted from the empirical production data. 


\section{Empirical analysis}

\subsection{Impact of service level on the size of the capacity buffer}

Based on the simulation results, Fig. 7 shows increasing the desired service level $(S L)$ inflates the size of capacity buffer nonlinearly and consequently squeezes the level of target house completion (THC).

Fig. 7. Optimal size of the capacity buffer in simulation experiments (enforcing a growing service level)

As Fig.7 reveals, increasing the service level decreases the level of target house completion (THC) in a nonlinear trend. That is, if the builder tends to have a reliable production and achieve on time completions, a conservative THC level should be maintained. This is consistent with the optimization results in the previous section (table 2) and provides a measure of validation. Furthermore, it extends finding of Arashpour, Wakefield et al. (2013), indicating that loading operations to the full capacity is not necessarily the best production strategy and a decent-sized capacity buffer will help both homebuilders by avoiding late completion costs and homebuyers by shortening the preoccupancy period.

\subsection{Impacts of the gross production capacity and workflow stability on the size of the capacity buffer}

Optimization models in the previous stage of the framework revealed that size of the capacity buffer is dependent on the average production capacity and standard deviation of time between completions. In a controlled simulation experiment, the gross production capacity fluctuated between 400 and 600 houses per year while the standard deviation of time between completions was controlled. Results of running the simulation experiment have been illustrated in Fig. 8a.

Fig. 8a. Target house completion (controlled for production variability)

Fig. 8b. Capacity buffer vs. production variability

Fig. 8. Simulation results on Target house completion and size of the capacity buffer

As can be seen in Fig. 8a, increasing the gross production capacity results in a growing level of target house completion (THC). However, it is controlling the standard deviation of time between completions that keeps the size of the capacity buffer constant. It is worth mentioning that having a stable workflow by fully controlling the variability over a long-term production is a difficult task, which is hardly achievable in construction sites. Therefore, the production variability should also be taken into consideration before loading the network of trades to full capacity and starting as many new constructions as possible, which is a common approach in the house building environment (Arashpour and Arashpour 2011).

Given the presence of variability, the number of unfinished jobs grows exponentially and the production system soon becomes unstable. Fig. 8b plots the increasing size of the capacity buffer against standard deviations of time between completions, which measure the workflow stability. As can be seen in this figure, construction production networks with more stable workflows require smaller capacity buffers. That is, the builder is able to meet the production target easier and consequently a higher service level is 
achievable. The striking difference between Fig. 8a and Fig. 8b highlights the high sensitivity of the capacity buffer size to the level of workflow stability. This is in line with the findings of González, Alarcón et al. (2011), indicating that focusing solely on capacity of the trade network can be misleading for builders and causes a lack of coordination between construction output and start. In fact, without the aid of an optimal capacity buffer, target house completion (THC) and expected profit will decline over the long-term production. This is consistent with the results of running optimization models in table 3 and validates them.

\section{Limitations}

Although using the proposed framework resulted in significant improvements in performance measures of the two case studies, a number of important limitations should be mentioned:

Firstly, using predictive models in the proposed framework was found plausible in the residential construction settings. This sector of the construction industry is very similar to manufacturing and future production can be predicted by analysing the past performance. Future work could test applicability of the framework in other construction subsectors.

Secondly, adopting two optimization models developed in the current research resulted in finding optimal (or near optimal) capacity buffers for the two case studies. However, this should be taken into consideration that these models are simplified to be easily used. For example, they assume that processing times are normally distributed, which is not always true in the real-world construction. Such assumptions, however, were not used in the simulation experiments at the fourth stage of the framework.

Thirdly, 1200 simulation experiments that were designed and analysed in this research are reflective of typical production scenarios in house building but are not comprehensive of every problem that could happen in construction sites. This should be taken into consideration that every construction project has its unique production environment and stochastic variables to be modelled.

\section{Conclusions}

Prior work has documented the effectiveness of rate-driven construction in improving tangible performance measures in construction projects (Choi and Minchin Jr 2006). Rate-driven construction, however, does not consider due date integrity explicitly and an additional control measure in form of a capacity buffer is required. Existing research in the construction literature in order to investigate tangible effects of capacity buffers on production metrics and the optimal size for such buffers is sparse. To bridge this gap, this study tested a user-friendly framework for finding the optimal size for capacity buffer that maximises the workflow stability and minimises the probability of late completions. Towards this aim, production data of two Australian construction companies were collected and analysed. Gross production capacity of the network was computed by using time series analysis. Then, cost and capacity optimizations were conducted to find the optimal size of the capacity buffer. Following this, results of mathematical modelling were linked to a discrete-event simulation engine and different real-life production scenarios caused by varying stochastic variables of construction production were analysed. 
The robustness of the framework in order to improve workflow stability through establishing a capacity buffer was tested. Findings show that an optimal-sized buffer can help construction systems in maintaining a synchronised production in which output and demand are coordinated. These findings extend those of Nasir, Haas et al. (2012) and Ballard (2000), confirming the positive impact of reducing and buffering variability on improving the productivity in construction. In addition, the results show that setting the optimal capacity buffer requires making trade-offs between lost revenue opportunity caused by big buffers and late completion costs caused by small capacity buffers.

\section{Research contributions and opportunities for extensions}

This work contributes to the body of knowledge by developing a deeper understanding of the role of capacity buffers in improving workflow stability in the construction production. The proposed framework avoids oversized buffers since they are wasteful, hinder performance, and disrupt the workflow. Furthermore, using the framework does not create undersized buffers as they increase the risk of late completions and a poor service level. The user-friendly framework is intended to assist builders in finding the most cost-effective way to operate their network of trade contractors. The authors are currently working on more sophisticated optimization models that consider more stochastic variables in real-world construction settings.

The framework was used at the project level in order to compute the gross production capacity and then the optimal capacity buffer for the entire construction network. It is recommended that the framework is also used at the specialty contractor level so as to compute the optimal capacity buffer for each single trade.

\section{Acknowledgements}

This paper is based upon work supported in part by the RMIT School of Graduate Research, through HDRPG 2014 Grant. Opinions expressed are those of the writers and not necessarily those of RMIT University. The authors gratefully acknowledge the contribution of Monarch Building Systems, GCS Construction and Dr John McAndrew at RMIT University to this research project.

\section{References}

AbouRizk, S., D. Halpin, Y. Mohamed and U. Hermann (2011). "Research in modeling and simulation for improving construction engineering operations." Journal of Construction Engineering and Management 137(10): 843.

Arashpour, M. and M. Arashpour (2010). "Gaining the Best Value from HR in Construction Companies." Proceedings of the 6th European Conference on Management Leadership and Governance: 23-33.

Arashpour, M. and M. Arashpour (2011). "Important factors influencing personnel performance of construction companies." Economics, Business and Management 2: 32-37.

Arashpour, M., M. Shabanikia and M. Arashpour (2012). "Valuing the contribution of knowledgeoriented workers to projects: a merit based approach in the construction industry." Australasian Journal of Construction Economics and Building 12(4): 1- 12.

Arashpour, M., R. Wakefield and N. Blismas (2013). Improving construction productivity: implications of even flow production principles. CIB World Building Congress 2013: Construction and Society, Queensland University of Technology. 
Arashpour, M., R. Wakefield and N. Blismas (2013). Role of simulation in construction processesharmony in capturing resources. Research, Development and Practice in Structural Engineering and Construction (ASEA-SEC), Research Publishing Services (RPS).

Arashpour, M., R. Wakefield, N. Blismas and E. W. M. Lee (2013). "A new approach for modelling variability in residential construction projects." Australasian Journal of Construction Economics and Building 13(2): 83-92.

Arashpour, M., R. Wakefield, N. Blismas and E. W. M. Lee (2014). "Analysis of disruptions caused by construction field rework on productivity in residential projects." Journal of Construction Engineering and Management 140(2).

Ballard, G. and L. Koskela (2009). Design should be managed as a physical process, too. 17th International Conference on Engineering Design, ICED 09, Palo Alto, CA.

Ballard, H. G. (2000). The last planner system of production control, PhD dissertation, University of Birmingham.

Bashford, H. H., A. Sawhney, K. D. Walsh and K. Kot (2003). "Implications of even flow production methodology for U.S. housing industry." Journal of construction engineering and management 129(3): 330-337.

Blair, A. N., L. M. Lye and W. J. Campbell (1993). "Forecasting construction cost escalation." Canadian journal of civil engineering 20(4): 602-612.

Chanmeka, A., S. R. Thomas, C. H. Caldas and S. P. Mulva (2012). "Assessing key factors impacting the performance and productivity of oil and gas projects in Alberta." Canadian Journal of Civil Engineering 39(3): 259-270.

Choi, J. and R. E. Minchin Jr (2006). "Workflow management and productivity control for asphalt pavement operations." Canadian Journal of Civil Engineering 33(8): 1039-1049.

Choy, E. and J. Y. Ruwanpura (2006). "Predicting construction productivity using situation-based simulation models." Canadian Journal of Civil Engineering 33(12): 1585-1600.

Dai, J. and P. M. Goodrum (2012). "Generational differences on craft workers' perceptions of the factors affecting labour productivity." Canadian Journal of Civil Engineering 39(9): 1018-1026.

Damrianant, J. and R. R. Wakefield (2000). "An alternative approach for modeling of interference in discrete-event systems." Civil Engineering and Environmental Systems 17(3): 213-235.

Dissanayake, M. and A. R. Fayek (2008). "Soft computing approach to construction performance prediction and diagnosis." Canadian Journal of Civil Engineering 35(8): 764-776.

Fayek, A. R., M. Dissanayake and O. Campero (2004). "Developing a standard methodology for measuring and classifying construction field rework." Canadian Journal of Civil Engineering 31(6): 1077-1089.

Georgy, M. E. (2008). "Evolutionary resource scheduler for linear projects." Automation in Construction 17(5): 573-583.

Goldratt, E. M. and J. Cox (2005). "A Process of Ongoing Improvement." Journal of Manufacturing Technology Management 16(3): 302.

González, V., L. Alarcón, S. Maturana and J. Bustamante (2011). "Site Management of Work-in-Process Buffers to Enhance Project Performance Using the Reliable Commitment Model: Case Study." Journal of construction engineering and management 137(9): 707-715.

Han, S., T. Hong, G. Kim and S. Lee (2011). "Technical comparisons of simulation-based productivity prediction methodologies by means of estimation tools focusing on conventional earthmovings." Journal of Civil Engineering and Management 17(2): 265-277.

Hazini, K., R. Dehghan and J. Ruwanpura (2013). "A heuristic method to determine optimum degree of activity accelerating and overlapping in schedule compression." Canadian Journal of Civil Engineering 40(4): 382-391.

Hegazy, T. and W. Menesi (2012). "Enhancing the critical path segments scheduling technique for project control." Canadian Journal of Civil Engineering 39(9): 968-977. 
Hewage, K. N., A. Gannoruwa and J. Y. Ruwanpura (2011). "Current status of factors leading to team performance of on-site construction professionals in Alberta building construction projects." Canadian Journal of Civil Engineering 38(6): 679-689.

Hopp, W. J. and M. L. Spearman (2008). Factory physics, McGraw-Hill Irwin Irwin.

Hopp, W. J., M. L. Spearman and I. Duenyas (1993). "Economic production quotas for pull manufacturing systems." IIE Transactions (Institute of Industrial Engineers) 25(2): 71-79.

Horman, M. J. and H. R. Thomas (2005). "Role of inventory buffers in construction labor performance." Journal of Construction Engineering and Management 131(7): 834-843.

Hsie, M., C. J. Chang, I. T. Yang and C. Y. Huang (2009). "Resource-constrained scheduling for continuous repetitive projects with time-based production units." Automation in Construction 18(7): 942-949.

Im, K. S., S. H. Han, B. Koo and D. Y. Jung (2009). "Formulation of a pull production system for optimal inventory control of temporary rebar assembly plants." Canadian Journal of Civil Engineering 36(9): 1444-1458.

Jarkas, A. M. (2010). "Buildability factors affecting formwork labour productivity of building floors." Canadian Journal of Civil Engineering 37(10): 1383-1394.

Kim, S. C. (2009). "Project success indicators focusing on residential projects: Are schedule performance index and cost performance index accurate measures in earned value?" Canadian Journal of Civil Engineering 36(11): 1700-1710.

Koskela, L., R. Sacks and J. Rooke (2012). A brief history of the concept of waste in production. 20th Conference of the International Group for Lean Construction, IGLC 2012, San Diego, CA.

Lander, E. and J. K. Liker (2007). "The Toyota Production System and art: Making highly customized and creative products the Toyota way." International Journal of Production Research 45(16): 36813698.

Leaman, A. and B. Bordass (1999). "Productivity in buildings: The 'killer' variables." Building Research and Information 27(1): 4-19.

Lee, E. W. M., I. W. H. Fung, V. W. Y. Tam and M. Arashpour (2013). "A fully autonomous kernelbased online learning neural network model and its application to building cooling load prediction." Soft Computing: 1-16.

Lee, J. S. and J. E. Diekmann (2011). "Delay analysis considering production rate." Canadian Journal of Civil Engineering 38(2): 361-372.

Moselhi, O., D. Gong and K. El-Rayes (1997). "Estimating weather impact on the duration of construction activities." Canadian Journal of Civil Engineering 24(3): 359-366.

Nasir, H., C. T. Haas, J. H. Rankin, A. R. Fayek, D. Forgues and J. Ruwanpura (2012). "Development and implementation of a benchmarking and metrics program for construction performance and productivity improvement." Canadian Journal of Civil Engineering 39(9): 957-967.

Sezer, A. A. and J. Bröchner (2013). "The construction productivity debate and the measurement of service qualities." Construction Management and Economics: 1-10.

Shahin, A., S. M. Abourizk, Y. Mohamed and S. Fernando (2014). "Simulation modeling of weathersensitive tunnelling construction activities subject to cold weather." Canadian Journal of Civil Engineering 41(1): 48-55.

Son, J., M. Mack and K. G. Mattila (2006). "Nonlinear cash flow optimization model." Canadian Journal of Civil Engineering 33(11): 1450-1454.

Sonmez, R. (2007). "Impact of occasional overtime on construction labor productivity: Quantitative analysis." Canadian Journal of Civil Engineering 34(7): 803-808.

Thomas, S. R., S. H. Lee and H. S. Park (2008). "Design-information technology and performances in building and industrial projects." Canadian Journal of Civil Engineering 35(10): 1102-1113.

Tommelein, I., D. Riley and G. Howell (1999). "Parade Game: Impact of Work Flow Variability on Trade Performance." Journal of construction engineering and management 125(5): 304-310.

Zayed, T. M. and I. A. Nosair (2006). "Cost management for concrete batch plant using stochastic mathematical models." Canadian Journal of Civil Engineering 33(8): 1065-1074. 